\title{
Resting Weak Cells to Improve Battery Pack's Capacity Delivery via Reconfiguration
}

\author{
Liang $\mathrm{He}$ \\ University of Michigan \\ 2260 Hayward St. \\ Ann Arbor, Michigan, 48109 \\ lianghe@umich.edu
}

\author{
Eugene Kim \\ University of Michigan \\ 2260 Hayward St. \\ Ann Arbor, Michigan, 48109 \\ kimsun@umich.edu
}

\author{
Kang G. Shin \\ University of Michigan \\ 2260 Hayward St. \\ Ann Arbor, Michigan, 48109 \\ kgshin@umich.edu
}

\begin{abstract}
Cell imbalance commonly found in large battery packs degrades their capacity delivery, especially for cells connected in series where the weakest cell dominates their overall capacity. In this paper, we present a case study of exploiting system reconfiguration to mitigate the cell imbalance in battery packs. Specifically, instead of using all the cells in a battery pack to support the load, selectively skipping cells to be discharged may actually enhance the pack's capacity delivery. Based on this observation, we propose CSR, a Cell Skipping-assisted Reconfiguration algorithm that identifies the system configuration with (near)-optimal capacity delivery. We evaluate CSR using large-scale emulation based on empirically collected discharge traces of 40 Lithium-ion cells. CSR is shown to achieve close-to-optimal capacity delivery when the cell imbalance in the battery pack is low and improve the capacity delivery by up to $94 \%$ in case of high imbalance.
\end{abstract}

\section{CCS Concepts}

-Computer systems organization $\rightarrow$ Embedded software;

\section{Keywords}

Reconfigurable battery packs, cell skipping, cell imbalance

\section{INTRODUCTION}

The ability to provide high and reliable power supply has made large battery packs widely used in systems such as power grids $[14,15,22]$ and electric vehicles (EVs) [28, 31]. For example, 7, 104 cells are used in Tesla Model S to power the vehicle with $85 \mathrm{kWh}[8]$ capacity. However, these large number of cells in the battery pack create severe cell imbalance, a notorious but commonly found problem in battery packs. Cell imbalance represents the fact that the strength of cells in accepting/delivering capacity tends to diverge

Permission to make digital or hard copies of all or part of this work for personal or classroom use is granted without fee provided that copies are not made or distributed for profit or commercial advantage and that copies bear this notice and the full citation on the first page. Copyrights for components of this work owned by others than the author(s) must be honored. Abstracting with credit is permitted. To copy otherwise, or republish, to post on servers or to redistribute to lists, requires prior specific permission and/or a fee. Request permissions from permissions@acm.org.

e-Energy'16, June 21-24, 2016, Waterloo, ON, Canada

(C) 2016 Copyright held by the owner/author(s). Publication rights licensed to ACM. ISBN 978-1-4503-4393-0/16/06 . .\$15.00

DOI: http://dx.doi.org/10.1145/2934328.2934336 over time and usage, caused by various uncontrollable factors such as manufacturing variability and operational thermal conditions. The unbalanced cells degrade their capacity delivery, especially for those connected in series (i.e., cell strings) - the cell string is only as strong as its weakest cell $[19,23]$. Also, the imbalance among cells easily leads to their over-charge/discharge, accelerating their capacity fading [20] and causing safety risks, such as thermal runaway [12].

Recently, reconfigurable battery packs, with their ability to dynamically alter the cell connectivity (e.g., in series or in parallel) and thus offering a new dimension for system improvement, have been receiving considerable attention $[13,16,18,25,26,30]$ and generating funding opportunities [6]. For example, the physical design of low-complexity reconfigurable battery packs has been explored in [23,24,26], and the trade-off between cycle efficiency and capacity utilization has been explored in [27].

The system reconfigurability can also be exploited to mitigate the cell imbalance in battery packs. In this paper, we present a case study of leveraging system reconfiguration to mitigate the cell imbalance and thus enhance the battery pack's capacity delivery. Specifically, focusing on the JPLtype reconfigurable battery packs [11], we aim to identify the system configuration with the maximum capacity delivery. We abstract this problem to optimally selecting cells in the battery pack with the observation that selectively skipping cells to be discharged - thus resting certain cells - may actually improve the battery pack's capacity delivery over the common approach of using all cells to power the load. Then we propose a Cell Skipping-assisted Reconfiguration (CSR) algorithm that identifies a (near)-optimal system configuration based on cells' real-time deliverable capacity via dynamic programming (DP) - CSR achieves (i) the maximum capacity delivery when the cells are ideal (i.e., when their rate capacity effect is negligible) and (ii) a bounded ratio to the maximum capacity delivery for cells with pronounced rate capacity effect. We also show that CSR reduces the cell imbalance in the long run and is not confined to JPL-type battery packs. This paper makes the following contributions.

- We present the first case study of exploiting system reconfiguration to mitigate cell imbalance in battery packs.

- With two empirically observed sequential properties of cells in the battery pack - one is imposed by the physical direction of discharge current and the other is 


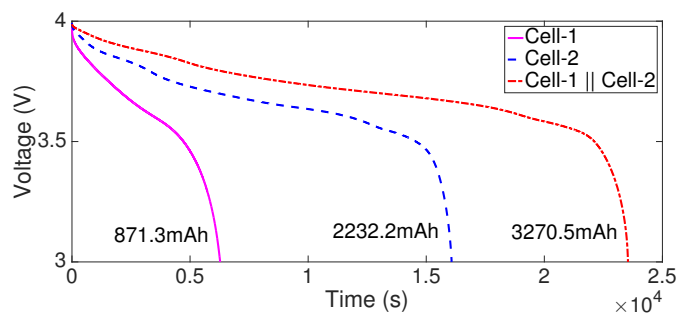

Figure 1: Parallel connection delivers the sum of string capacities.

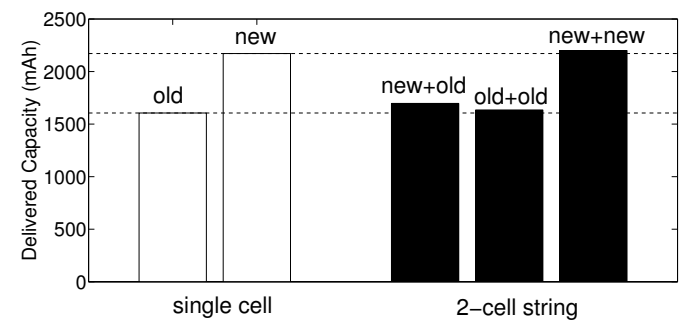

Figure 2: Capacity delivery of cell string is dominated by the weakest cell.

to avoid the physical short of cells — we abstract the problem of identifying the system configuration with maximum capacity delivery to a cell-selection problem in the battery pack.

- We design CSR, a reconfiguration algorithm that identifies the system configuration with (near)-optimal capacity delivery.

- We evaluate CSR with emulation based on the discharge traces of 40 Lithium-ion cells, demonstrating up to $94 \%$ improvement in capacity delivery, especially when facing high cell imbalance. This improvement is significant in view of the fact that the battery density has only doubled over the past 15 years [4].

The paper is organized as follows. Sec. 2 introduces the basic concepts related to battery cells and reconfigurable battery packs. We present the problem abstraction and our motivating observation in Secs. 3 and 4. CSR is introduced in Sec. 5, its salient properties are discussed in Sec. 6, and the evaluation results are provided in Sec. 7. Sec. 8 reviews the related work. The paper concludes with Sec. 9.

\section{BACKGROUND AND SYSTEM MODEL}

Below we introduce the background and system model.

\subsection{Cell Basics}

- $C$-Rate of Cells. The discharge current of cells is often expressed as C-rate [1]. Specifically, C-rate is a measure of the rate at which the cell is discharged relative to its rated capacity - a $1 \mathrm{C}$ rate means the discharge current will drain the cell completely in 1 hour. For example, the $1 \mathrm{C}$ rate for a cell with $2,900 \mathrm{mAh}$ rated capacity equates to a discharge current of $2,900 \mathrm{~mA}$, and a $2 C$ rate would be $5,800 \mathrm{~mA}$.

- Cell Connectivity and Cell Imbalance. Cells are the basic units of a battery pack. The connectivity among cells determines the battery pack's output voltage and its

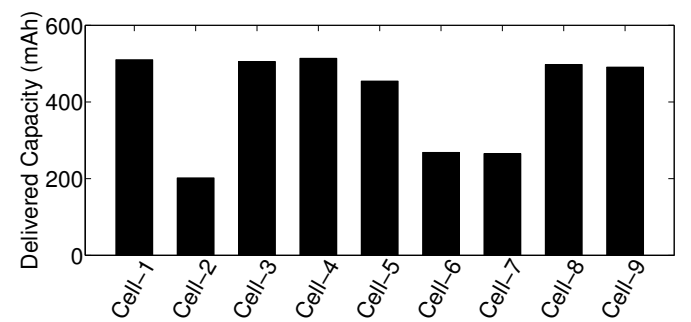

Figure 3: Delivered capacity of 9 cells purchased in the same batch.

capacity delivery. In general, cells in a battery pack can be connected in series or in parallel. The series connection of cells (i.e., a cell string) supplies a voltage that is the sum of individual cells. Cells connected in series have the same discharge current, and thus the weakest cell dominates their overall capacity delivery. On the other hand, connecting multiple cell strings in parallel does not increase the supplied voltage but splits the discharge current among the strings. The deliverable capacity of parallel strings is the sum of their respective deliverable capacities.

People may doubt this physical law on the capacity delivery of parallel strings when they are of various strength. As a validation, we connect two fully charged cells in parallel - i.e., form two parallel 1-cell strings, and discharge them with $500 \mathrm{~mA}$ current until a cut-off voltage of $3.0 \mathrm{~V}$ is reached. Fig. 1 plots the voltage trace during discharging ${ }^{1}$, together with those when discharging the two cells individually with the same current for comparison. The parallel connection delivers $3,270.5 \mathrm{mAh}$ capacity, which is roughly the sum of the two cells' individual capacity but a little larger (i.e., $3,270.5-(2,232.2+871.3)=167.0 \mathrm{mAh})$. This slightly increased capacity delivery can be explained with the rate-capacity effect because the discharge current of individual cells is reduced when connecting them in parallel, as we explain later.

The fact that the weakest cell of a cell string dominates its capacity delivery is a fundamental physical property inspiring this work. To clearly demonstrate this, we collect a set of measurements with four off-the-shelf $2,300 \mathrm{mAh}$ cells - two of them have been in use over one year (and thus are weaker) and the other two are new (and thus are stronger). Fig. 2 plots the collected results when discharging these cells with $1 \mathrm{C}$ rate. The new cells deliver $2,171.3 \mathrm{mAh}$ capacity on average, while the old cells deliver only $1,605.6 \mathrm{mAh}$. Then we form three 2-cell strings (i.e., new-and-old, old-and-old, and new-and-new) and again discharge them with $1 \mathrm{C}$ rate. A clear observation is that the new-and-old string delivers similar capacity as the old cells, validating the string capacity is dominated by the weakest cell. This means not all the capacity of series connected cells can be effectively delivered due to the weak cells.

The diverse cell strength, known as the cell imbalance issue, widely exists in battery packs. We discharge 9 Lithiumion cells ${ }^{2}$ and record their delivered capacity to demonstrate the cell imbalance, as summarized in Fig. 3. Obvious difference is observed - the strongest cell (i.e., cell-4) delivers

\footnotetext{
${ }^{1}$ The operator $\|$ denotes the parallel connection of cell strings.

${ }^{2}$ These cells are of the same model and purchased in the same batch in 2013.)
} 


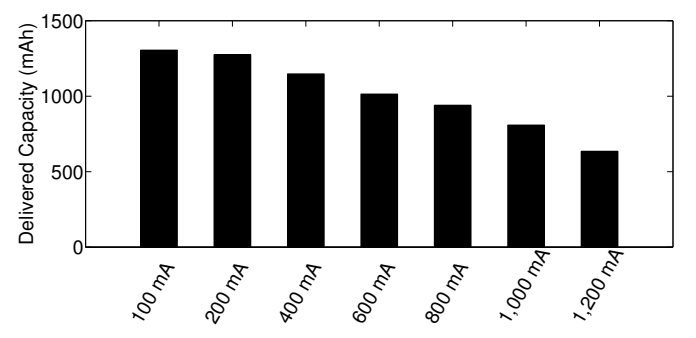

Figure 4: High discharge rates decrease cell's capacity delivery.

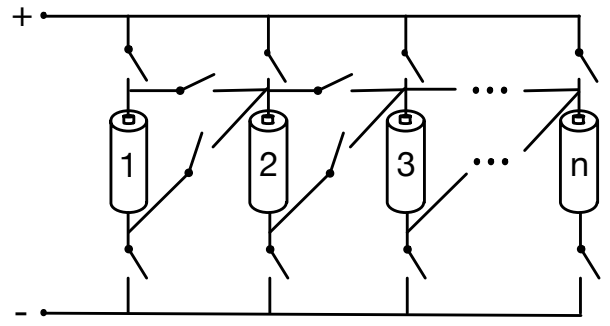

Figure 5: JPL-type reconfigurable battery packs [11].

$2.54 x$ capacity of the weakest one (i.e., cell-2). Even worse, conventional wisdom says the cell imbalance increases as cells age - their capacity delivery could vary as much as 10x for 5-year cells [17].

- Rate-Capacity Effect and Peukert's Law. Cells demonstrate nonlinear properties due to their electrochemical characteristics, such as the rate-capacity effect - the deliverable capacity of cells decreases with higher discharge rates. Fig. 4 plots our measurements when discharging a cell with currents from $100-1,200 \mathrm{~mA}$ - only $635.2 \mathrm{mAh}$ capacity is delivered with a discharge current of $1,200 \mathrm{~mA}$, about $48.7 \%$ of that when discharged with $100 \mathrm{~mA}$.

Peukert's Law [29] captures the rate-capacity effect with the basic form of $C=I^{\alpha} t$, where $C$ is the cell's rated capacity, $I$ is the discharge rate, $t$ is the actual discharge time, and $\alpha(\alpha \geq 1)$ is the Peukert coefficient capturing the cell's nonlinear property - an $\alpha$ of 1 reflects the ideal cells whose capacity delivery is independent to discharge rate (and thus the rate-capacity effect is negligible) and a larger $\alpha$ indicates a pronounced rate-capacity effect. Given rated capacity $C$ and the corresponding discharge rate $I$, Peukert's law can be extended to estimate cell's capacity delivery when discharged with $I^{\prime}$ as [5]

$$
C^{\prime}=C\left(I / I^{\prime}\right)^{\alpha-1}
$$

\subsection{JPL-Type Reconfigurable Battery Packs}

In contrast to traditional battery packs with fixed cell connectivity, reconfigurable battery packs $[16,23]$ offer a new dimension to improve the battery pack performance with the ability to alter the connectivity among cells (e.g., according to load requirements [18] and cell states [21]).

In this work, we present a case study of exploiting reconfiguration to improve the battery pack's capacity delivery. Specifically, we focus on JPL [11], a classic reconfigurable battery pack design as shown in Fig. 5, in which each cell is coupled with four switches. By controlling the close/open

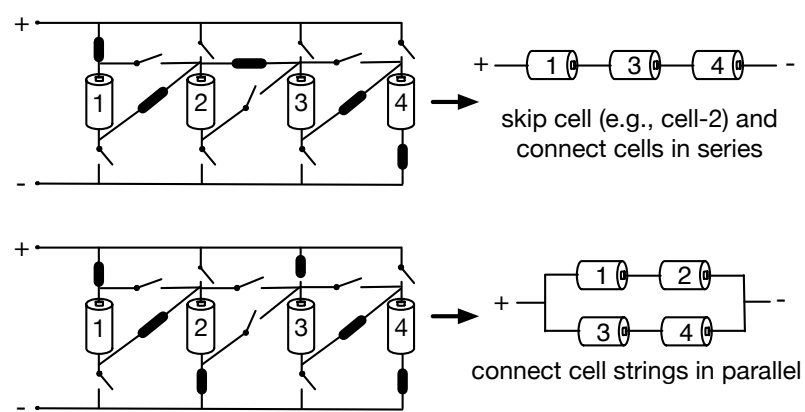

Figure 6: Adjusting cell connectivity by controlling the open/close states of switches.

states of these switches, we can skip cells from discharge, connect cells in series, and connect multiple cell strings in parallel, as illustrated in Fig. 6. We refer reconfigurable battery packs designed according to Fig. 5 as JPL-type battery packs for presentation convenience.

\subsection{System Model}

We consider the system model in Fig. 7, mainly consisting of the following components.

- Battery pack and load. A JPL-type battery pack consisting of $n$ cells is used to support load $\langle V, P\rangle$, where $V$ and $P$ are the load required voltage and power, respectively. Each cell in the pack has a nominal voltage $v$, and thus cell strings consisting of $m=\left\lceil\frac{V}{v}\right\rceil$ cells need to be formed to support the load.

- Diodes and regulators. Each cell string is connected with an diode to regulate the current direction, eliminating the potential safety issues, e.g., reverse charging, caused by the imbalance among multiple strings. Moreover, DC/DC converters are added between the battery pack and the load to ensure a stable voltage supply. ${ }^{3}$

- Battery management system (BMS). During individual charge/discharge cycles as shown in Fig. 8, the BMS monitors the real-time cell states, estimates their respective deliverable capacities, and identifies the proper system configuration to support the load. The thus-identified configuration is applied after charging the battery pack, which is then connected to the load and discharged - i.e., the battery pack is reconfigured offline.

Our goal is to design a reconfiguration algorithm for the BMS to identify the system configuration with the maximum capacity delivery, prolonging the load operation (e.g., extending the driving range of EVs).

Note that altering the system configuration during discharge (i.e., online reconfiguration) is possible in theory but in practice, the BMS should only reconfigure the system

\footnotetext{
${ }^{3}$ Supplying $V$ with cell strings of different sizes via DC/DC conversion is possible, but is of lower efficiency due to the larger difference between the supplied and required voltages $[18,32]$.
} 


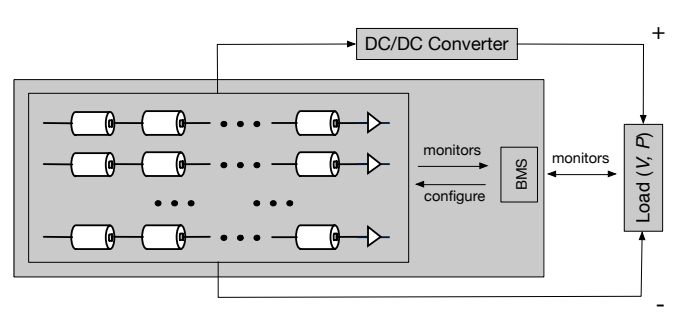

Figure 7: System model.

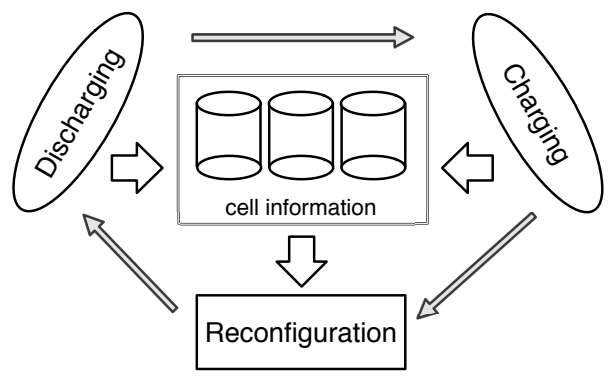

Figure 8: Application scenario.

when the load is disconnected and when proper safety protections are provided, leading to limited reconfiguration opportunities. This is because altering system configuration during discharge may cause safety risks such as arc flash due to voltage transients or loose connections [9], and the resultant inrush current could be as high as 50x of the normal current, severely jeopardizing system safety. Actually, a rule-of-thumb when reconfiguring electricity systems is to de-energize [10]. This way, we only consider such offline reconfiguration scenarios here.

Below we summarize the important notations used in this paper for the ease of reference.

- $n$ : the number of cells in the battery pack;

- $v$ : the nominal voltage of cells;

- $\alpha$ : Peukert's coefficient capturing the strength of the rate-capacity effect.

- $\langle V, P>$ : the load required voltage and power, indicating a required current of $\frac{P}{V}$ and a required cell string size of $m=\left\lceil\frac{V}{v}\right\rceil$;

- $c_{i}(i=1,2, \cdots, n)$ : the deliverable capacity of the $i$ th cell under $1 \mathrm{C}$ discharge rate;

- $C_{i}(i=1,2, \cdots, k)$ : the deliverable capacity of the $i$ th cell string under $1 \mathrm{C}$ discharge rate;

- $\mathbb{C}_{\text {ideal }}$ : the deliverable capacity of the battery pack with idealized cells, i.e., $\alpha=1$;

- $\mathbb{C}_{\mathrm{rc}}$ : the actual deliverable capacity of the battery pack when considering the rate-capacity effect, i.e., $\alpha>1$.

\section{PROBLEM ABSTRACTION}

In general, the problem of identifying the system configuration with maximum capacity delivery consisting of two

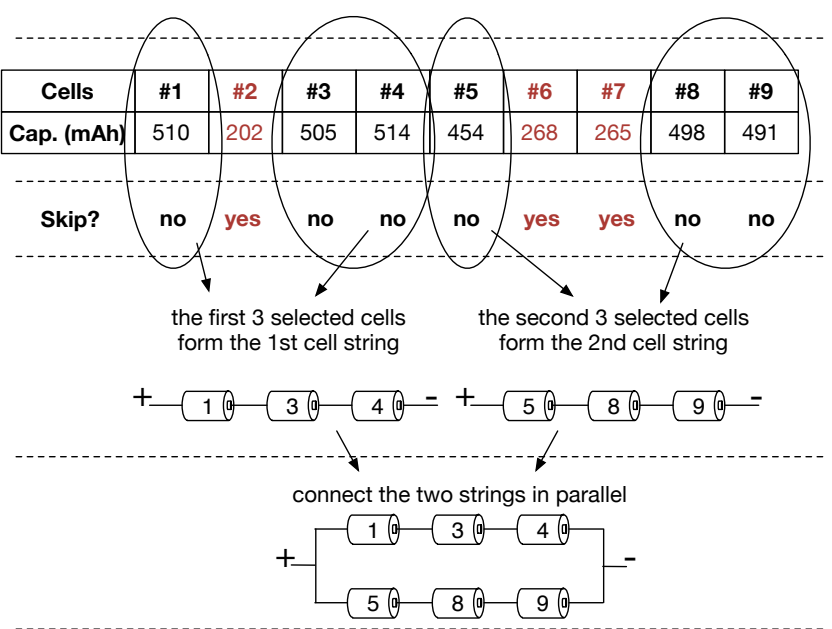

Figure 9: Illustrative example on abstracting the problem of identifying the optimal configuration to determining which cells to skip.

parts: (i) which cells should be used to support the load; (ii) how these cells should be connected. However, with the physical design of JPL-type battery packs, only the first question needs to be addressed and the answer to the second one follows straightforwardly, because of the sequential properties of cells in JPL-type battery packs.

Ascendingly indexing cells according to their physical distances to the output terminals as in Fig. 5, we observe the following two sequential properties shared by all legal system configurations of JPL-type battery packs. By legal system configuration, we mean (i) it is feasible for the battery pack to achieve such configuration; (ii) the battery packs can safely support the load with that configuration.

- Intra-String Sequential Property: for any legal cell string, the indexes of its cells are monotonic increasing. This is because the discharge current is directional and can only pass through cells with smaller indexes before those with larger indexes. Take the 4cell battery pack in Fig. 6 as an example, the cell string $\{1 \rightarrow 3 \rightarrow 2\}$ is not legal as the current cannot flow reversely from cell-3 to cell-2, indicating this string is not physically achievable.

- Inter-String Sequential Property: for any legal configuration with parallel cell strings, the indexes of cells in these strings are also monotonic increasing it is always feasible to index these strings as the 1 st string, the 2nd string, etc, such that for any $i<j$, the indexes of cells in the $i$ th string are smaller than those in the $j$ th string. This inter-string sequential property is to avoid shorting cells in the pack. Again, for the battery pack in Fig. 6, the configuration of $\{1 \rightarrow 3\} \|\{2 \rightarrow 4\}$ is not legal as cells in these two strings do not demonstrate monotonic increasing relationship - using these two strings simultaneously will short cell-2, albeit both of them are physically achievable.

These sequential properties indicate that (i) if a cell is skipped when forming the current string, it cannot be used to form other strings later, and thus its capacity cannot be 
used to support the load; (ii) the strings can only be formed sequentially with selected cells according to the increasing order of their indexes - the first $m$ selected cells form the 1st string, the second $m$ selected cells form the 2nd string, etc, where $m=\left\lceil\frac{V}{v}\right\rceil$ is the number of series cells required by the load.

These further lead to the following problem abstraction: for JPL-type battery packs, the problem of identifying the configuration with maximum capacity delivery is equivalent to optimally determining which cells should be used to support the load, after which the question of how to connect these selected cells can be answered accordingly. Fig. 9 shows an example on the problem abstraction with a JPLtype battery pack consisting of the 9 cells in Fig. 3 and the load requires 3-cell strings - if we decide to skip cell-2, cell-6, and cell-7 from discharge, the system configuration is also determined by sequentially forming the strings with remaining cells, i.e., $\{1 \rightarrow 3 \rightarrow 4\} \|\{5 \rightarrow 8 \rightarrow 9\}$.

\section{WHY TO SKIP CELLS?}

Intuitively, we want to use all the cells in the battery pack (thus forming as many parallel cell strings as possible) to support the load, especially in view of the rate-capacity effect - more parallel strings reduce the discharge rate of individual cells and thus improve their capacity delivery.

However, the widely existed cell imbalance, together with the fact that the weakest cell dominates the capacity delivery of a cell string, lead to the observation that sometimes selectively skipping cells to be discharged may actually improve the battery pack's capacity delivery. Let us again consider the JPL-type battery pack in Fig. 9, in which the deliverable capacities of cells under $1 \mathrm{C}$ discharge rate are listed. When all these 9 cells are used, we can form three 3 -cell strings to support the load in parallel: $\{1 \rightarrow 2 \rightarrow 3\} \|\{4 \rightarrow$ $5 \rightarrow 6\} \|\{7 \rightarrow 8 \rightarrow 9\}$ (Fig. 10). The deliverable capacity of these strings are $202 \mathrm{mAh}$ (dominated by cell-2), $268 \mathrm{mAh}$ (dominated by cell-6), and $265 \mathrm{mAh}$ (dominated by cell- 7 ), respectively. However, if we skip cell-2, cell-6, and cell-7 from discharge, we can form two cell strings $\{1 \rightarrow 3 \rightarrow 4\}$ and $\{5 \rightarrow 8 \rightarrow 9\}$ with a total deliverable capacity of $505+491=996 \mathrm{mAh}$, which is $35 \%$ more when compared with using all the cells (i.e., $202+268+265=735 \mathrm{mAh}$ ).

The problem becomes more tricky when considering the rate-capacity effect. Two strings are formed in the above example when cells are skipped, meaning each remaining cell needs to supply a current of $\frac{P}{2 V}$ to the load. On the other hand, three strings are formed when all cells are used, and thus each cell only needs to supply a current of $\frac{P}{3 V}$. By Peukert's law (Eq. (1)), we know the actual capacity delivery of the battery pack is $996 \times\left(2 V \cdot I_{C} / P\right)^{\alpha-1}$ when skipping cells and $735 \times\left(3 V \cdot I_{C} / P\right)^{\alpha-1}$ when using all the cells, where $I_{C}$ is the $1 \mathrm{C}$ discharge rate in Amps - there is no one-for-all answer to which one is larger without the information on $V, P$, and $\alpha$.

The above example reveals a dilemma when selecting cells to support the load: selectively skipping cells may increase the capacity delivery with ideal cells but forms less parallel strings, which on the other hand increases cells' discharge rate and thus degrades their capacity delivery due to the ratecapacity effect.

The three weakest cells in the pack are skipped from discharge to facilitate the illustration in the example shown in Fig. 10. In practice, however, greedily skipping the weakest

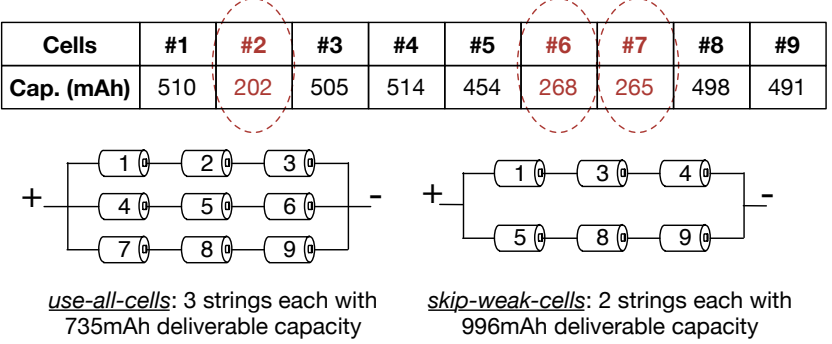

Figure 10: Illustrative example: skipping cells from discharge may improve the battery pack's capacity delivery.

cells is not always the best solution, as we will see in Sec. 7 .

\section{CELL SKIPPING-ASSISTED RECONFIG- URATION}

Denote cells' deliverable capacity under $1 \mathrm{C}$ discharge rate as $\left\langle c_{1}, c_{2}, \cdots, c_{n}\right\rangle\left(c_{i}>0\right)$, which can be obtained from their previous discharge cycles. CSR uses a DP-based method to determine which, if any, cells should be skipped from discharge, thus identifying the configuration with (near)optimal capacity delivery.

\subsection{DP-based Cell Skipping}

For the ease of description, let us first assume ideal cells (i.e., $\alpha=1$ ) in the battery pack, i.e., the rate-capacity effect is negligible. Define the cell skipping vector $S_{1 \times n}=$ $\left\{s_{i}\right\}(i=1,2, \cdots, n)$ as

$$
s_{i}= \begin{cases}1 & \text { if cell }-i \text { is skipped from discharge } \\ 0 & \text { otherwise. }\end{cases}
$$

From Sec. 3, we know any instance of $S_{1 \times n}$ also defines a system configuration. Furthermore, the sequential properties of cells allow us to identify the optimal configuration of an $n$ cell battery pack $S_{1 \times n}^{*}$ based on the optimal configurations when considering only its first $(n-i)(i=1,2, \cdots, m-1)$ cells $S_{1 \times(n-i)}^{*}$ - the sub-optimal structure of DP.

Define $H_{m}(i, j)(j-i \geq m-2)$ as the largest $(m-1)$ elements among $\left\{c_{i}, c_{i+1}, \cdots, c_{j}\right\}$. Further define $g_{m}(i, j)$ as the deliverable capacity of the string formed by the $j$-th cell and the cells corresponding to $H_{m}(i, j)$. Specifically,

$$
\begin{aligned}
g_{m}(i, j)= & \min \left\{H_{m}(i, j-1), c_{j}\right\} \\
& (i=1,2, \cdots n-m+1 ; \\
& j=m+i-1, m+i, \cdots, n) .
\end{aligned}
$$

Define $f_{m}(j)(j=1,2, \cdots, n)$ as the maximum deliverable capacity when only considering the first $j$ cells in the pack to support the load and cell- $j$ is not skipped (i.e., $s_{j}=0$ ), meaning cell- $j$ is the last cell of a $m$-cell string. Clearly,

$$
f_{m}(1)=f_{m}(2)=\cdots=f_{m}(m-1)=0 .
$$

Further defining $f_{m}(0)=0$, we have the following optimal substructure based on which the system configuration with maximum deliverable capacity can be identified

$$
\begin{aligned}
f_{m}(j)= & \max \left\{f_{m}(i)+g_{m}(i+1, j)\right\} \\
& (j=m, m+1, \cdots, n ; \quad i=0,1, \cdots, j-m) .
\end{aligned}
$$

With ideal cells in the battery pack, its maximum deliverable capacity is $\mathbb{C}_{\text {ideal }}^{\mathrm{dp}}=\max \left\{f_{m}(j)\right\}$, and the corresponding 


\begin{tabular}{|c|c|c|c|c|c|c|c|c|c|}
\hline$g_{m=3}(i, j)$ & $j=1$ & $j=2$ & $j=3$ & $j=4$ & $j=5$ & $j=6$ & $j=7$ & $j=8$ & $j=9$ \\
\hline$\overline{i=1}$ & - & - & $202(1,2,3)$ & $\overline{c 505(1,3,4)}$ & $\overline{4454(1,4,5)}$ & $268(1,4,6)$ & $265(1,4,7)$ & $4498(1,4,8)$ & $4991(1,4,9)$ \\
\hline$i=2$ & - & - & - & $202(2,3,4)$ & $454(3,4,5)$ & $268(3,4,6)$ & $265(3,4,7)$ & $498(3,4,8)$ & $491(3,4,9)$ \\
\hline$i=3$ & - & - & - & - & $454(3,4,5)$ & $268(3,4,6)$ & $265(3,4,7)$ & $498(3,4,8)$ & $491(3,4,9)$ \\
\hline$i=4$ & - & - & - & - & - & $268(4,5,6)$ & $265(4,5,7)$ & $454(4,5,8)$ & $491(4,8,9)$ \\
\hline$i=5$ & - & - & - & - & - & - & $265(5,6,7)$ & $268(5,6,8)$ & $454(5,8,9)$ \\
\hline$i=6$ & - & - & - & - & - & - & - & $265(6,7,8)$ & $268(6,7,9)$ \\
\hline$i=7$ & - & - & - & - & - & - & - & - & $265(7,8,9)$ \\
\hline$i=8$ & - & - & - & - & - & - & - & - & - \\
\hline$i=9$ & - & - & - & - & - & - & - & - & - \\
\hline$f_{m=3}(j)$ & $0(-,-)$ & $0(-,-)$ & $202(-, g(1,3))$ & $505(-, g(1,4))$ & $454(3, g(3,5))$ & $470(4, g(4,6))$ & $770(4, g(5,7))$ & $773(4, g(5,8))$ & $959(4, g(5,9))$ \\
\hline
\end{tabular}

Figure 11: A walk-through example on $\operatorname{CSR}(n=9, m=3)$.

configuration $S_{1 \times n}^{\mathrm{dp}}$ can be identified via reversing the search from $j^{\mathrm{dp}}=\max _{j}\left\{f_{m}(j)\right\}$.

\subsection{Walk-Through Example}

Next we use a walk-through example based on the 9-cell battery pack in Fig. 10 to facilitate the understanding of the DP-based cell skipping. Let us first consider $g_{m=3}(i, j)$. For example, when $i=2$ and $j=7, g_{m=3}(2,7)$ returns the capacity of the string formed by the $m-1=2$ cells from cell- 2 to cell- $(7-1)=6$ with the maximum deliverable capacity (i.e., $H_{m=3}(2,6)$ ), and with cell- 7 as the last cell. Specifically,

$$
g_{m=3}(2,7)=\min \left\{H_{m=3}(2,6), \quad c_{7}\right\} .
$$

As $H_{m=3}(2,6)$ is the largest two elements among

$$
\left\{c_{2}, c_{3}, \cdots, c_{6}\right\}=\{202,505,514,454,268\},
$$

we know $H_{m=3}(2,6)=\{505,514\}$ and

$$
\begin{aligned}
g_{m=3}(2,7) & =\min \{505,514,265\} \\
& =265 .
\end{aligned}
$$

Other $g_{m=3}(i, j)$ s can be calculated similarly as summarized in Fig. 11. Fig. 11 also lists the corresponding selected cells for each $g_{m=3}(i, j) \mathrm{s}$. For example,

$$
g_{m=3}(2,7)=265(3,4,7)
$$

means cell-3, cell-4, and cell-7 are selected to form the string, delivering $265 \mathrm{mAh}$ capacity. This way, $f_{m=3}(j) \mathrm{s}$ can be iteratively calculated according to Eq. (2). For example,

$$
\begin{aligned}
f_{m=3}(4) & =\max \left\{\begin{array}{c}
f_{m=3}(0)+g_{m=3}(1,4), \\
f_{m=3}(1)+g_{m=3}(2,4)
\end{array}\right\} \\
& =\max \{202,505\}=505, \\
f_{m=3}(5) & =\max \left\{\begin{array}{c}
f_{m=3}(0)+g_{m=3}(1,5), \\
f_{m=3}(1)+g_{m=3}(2,5), \\
f_{m=3}(2)+g_{m=3}(3,5)
\end{array}\right\} \\
& =\max \{454,454,454\} \\
& =454,
\end{aligned}
$$

Other $f_{m=3}(j) \mathrm{s}$, together with how they are obtained, are also summarized in Fig. 11. For example,

$$
f_{m=3}(8)=773(4, g(5,8))
$$

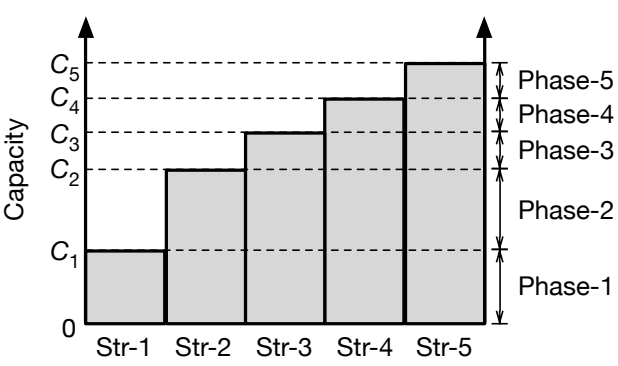

Figure 12: The discharge process can be divided into $k$ phases, where $k$ is the number of parallel strings and $k=5$ in this example.

means $f_{m=3}(8)$ is obtained based on $f_{m=3}(4)$ and $g_{m=3}(5,8)$. As $f_{m=3}(9)=959$ is the maximum of $f_{m=3}(j) \mathrm{s}$, we know the configuration with maximum capacity uses cell- 9 as the last cell. This way, we reverse the search from $f_{m=3}(9)$ and find

$$
\begin{aligned}
f_{m=3}(9) & =f_{m=3}(4)+g_{m=3}(5,9) \\
& =g_{m=3}(1,4)+g_{m=3}(5,9) .
\end{aligned}
$$

From Fig. 11, we know cell-1, cell-3, and cell-4 are used to form the string of $g_{m=3}(1,4)$, while those for $g_{m=3}(5,9)$ are cell-5, cell-8, and cell-9, indicating an identified configuration of

$$
\{1 \rightarrow 3 \rightarrow 4\} \|\{5 \rightarrow 8 \rightarrow 9\},
$$

and a skipping vector

$$
S_{1 \times 9}^{\mathrm{dp}}=\{0,1,0,0,0,1,1,0,0\} .
$$

The DP-based cell skipping requires a space complexity of $\mathcal{O}\left(n^{2}\right)$ and a computation complexity of $\mathcal{O}\left(m n^{2} l g n\right)$ - both dominated by the space/computation to store/calculate $g_{m}(i, j) \mathrm{s}$.

\subsection{Near-Optimality Analysis}

CSR identifies the configuration with maximum capacity delivery for ideal cells (i.e., $\alpha=1$ ). Below we prove its bounded near-optimality in capacity delivery even for cells with $\alpha>1$.

First, we have the following lemma on the capacity delivery of a given configuration when considering the ratecapacity effect. Note this is also the capacity delivery for non-reconfigurable battery packs.

Lemma 1. For any configuration with $k\left(1 \leq k \leq\left\lfloor\frac{n}{m}\right\rfloor\right)$ parallel $m$-cell strings, denote the deliverable capacity of 
these $k$ strings (in ascending order) under $1 \mathrm{C}$ discharge as

$$
C_{1} \leq C_{2} \leq \cdots \leq C_{k}
$$

and further define $\mathbb{C}_{\text {ideal }}=\sum_{i=1}^{k} C_{i}$. When using this configuration to support load $\langle V, \bar{P}\rangle$, its actual deliverable capacity $\mathbb{C}_{\mathrm{rc}}$ when considering the rate-capacity effect is

$$
\mathbb{C}_{\mathrm{rc}}=\left(V \cdot I_{C} / P\right)^{\alpha-1} \sum_{i=1}^{k}(k-i+1)^{\alpha}\left(C_{i}-C_{i-1}\right) .
$$

where $I_{C}$ is the $1 \mathrm{C}$ discharge current in Amps.

Proof. The entire discharge process can be divided into $k$ phases when these $k$ strings are connected in parallel to support the load, as illustrated in Fig. 12.

- Phase-1: The Phase-1 of the discharge process starts when the discharge begins and ends when the weakest cell string (i.e., the one with deliverable capacity $C_{1}$ ) depletes. During this phase, the load is supported with $k$ parallel strings, and each of the string has a discharge rate of $I_{1}=\frac{P}{k \cdot V}$. By Peukert's law, ${ }^{4}$ the delivered capacity during this phase is

$$
k \cdot C_{1}\left(I_{C} / I_{1}\right)^{\alpha-1} \text {. }
$$

- Phase-2: This discharge phase starts from the depletion of the weakest string and ends when the second weakest string (i.e., the one with deliverable capacity $\left.C_{2}\right)$ is drained. Only $(k-1)$ parallel strings are available to support the load during this phase, leading to a discharge rate of $I_{2}=\frac{P}{(k-1) \cdot V}$ for each string. This way, the delivered capacity during Phase-2 is

$$
(k-1) \cdot\left(C_{2}-C_{1}\right)\left(I_{C} / I_{2}\right)^{\alpha-1} .
$$

$\bullet \cdots \cdots$

- Phase- $k$ : The last discharge phase is when only the strongest cell string (i.e., the one with deliverable capacity $C_{k}$ ) is available to support the load. During this phase, the strongest string has a discharge rate of $I_{k}=\frac{P}{1 \cdot V}$ and the delivered capacity is

$$
1 \cdot\left(C_{k}-C_{k-1}\right)\left(I_{C} / I_{k}\right)^{\alpha-1}
$$

Further defining $C_{0}=0$, the deliverable capacity of the configuration when considering the rate-capacity is

$$
\begin{aligned}
\mathbb{C}_{\mathrm{rc}} & =\sum_{i=1}^{k}(k-i+1)\left(C_{i}-C_{i-1}\right)\left(I_{C} / I_{i}\right)^{\alpha-1} \\
& =\left(V \cdot I_{C} / P\right)^{\alpha-1} \sum_{i=1}^{k}(k-i+1)^{\alpha}\left(C_{i}-C_{i-1}\right) .
\end{aligned}
$$

Lemma 1 in turn leads to the following lemma on the maximum deliverable capacity $\mathbb{C}_{\mathrm{rc}}$ of any configuration with given $\mathbb{C}_{\text {ideal }}$.

Lemma 2. For any configuration with given $\mathbb{C}_{\text {ideal }}$,

$$
\mathbb{C}_{\mathrm{rc}} \leq \mathbb{C}_{\text {ideal }}\left(k \cdot V \cdot I_{C} / P\right)^{\alpha-1} .
$$

\footnotetext{
${ }^{4}$ Note that both Peukert's law and Peukert coefficient are only to analytically track CSR's (near)-optimality, but are not required when implementing CSR.
}

Furthermore, when $\alpha>1$, the equality holds iff

$$
C_{1}=C_{2}=\cdots=C_{k}=\mathbb{C}_{\text {ideal }} / k .
$$

Proof. It is clear that $\mathbb{C}_{\mathrm{rc}}=\mathbb{C}_{\text {ideal }}\left(k \cdot V \cdot I_{C} / P\right)^{\alpha-1}$ when $C_{1}=C_{2}=\cdots=C_{k}=\mathbb{C}_{\text {ideal }} / k$. To show that $\mathbb{C}_{\text {ideal }}\left(k \cdot V \cdot I_{C} / P\right)^{\alpha-1}$ is also the maximum capacity delivery with given $\mathbb{C}_{\text {ideal }}$, we define $\delta_{i}=C_{i}-C_{i-1}(i=$ $1,2, \cdots, k)$, and thus

$$
k \delta_{1}+(k-1) \delta_{2}+\cdots+\delta_{k}=\mathbb{C}_{\text {ideal }} .
$$

Denote $\mathbb{C}_{\mathrm{rc}}^{1}$ as the capacity delivery of the configuration with $C_{1}=C_{2}=\cdots=C_{k}=\mathbb{C}_{\text {ideal }} / k$, and $\mathbb{C}_{\mathrm{rc}}^{2}$ as the capacity delivery of any other configurations with $\mathbb{C}_{\text {ideal }}$, we have

$$
\begin{aligned}
& \mathbb{C}_{\mathrm{rc}}^{1}-\mathbb{C}_{\mathrm{rc}}^{2} \\
=\quad & \left(V \cdot I_{C} / P\right)^{\alpha-1} k^{\alpha-1} \mathbb{C}_{\text {ideal }}- \\
& \left(V \cdot I_{C} / P\right)^{\alpha-1} \sum_{i=1}^{k}(k-i+1)^{\alpha}\left(C_{i}-C_{i-1}\right) \\
= & \left(V \cdot I_{C} / P\right)^{\alpha-1}\left[k ^ { \alpha - 1 } \left(k \delta_{1}+(k-1) \delta_{2}+\cdots\right.\right. \\
& \left.\left.+\delta_{k}\right)-\left(k^{\alpha} \delta_{1}+(k-1)^{\alpha} \delta_{2}+\cdots+\delta_{k}\right)\right] \\
= & \left(V \cdot I_{C} / P\right)^{\alpha-1}\left[(k-1)\left(k^{\alpha-1}-(k-1)^{\alpha-1}\right) \delta_{2}\right. \\
& +(k-2)\left(k^{\alpha-1}-(k-2)^{\alpha-1}\right) \delta_{3}+ \\
& \left.\cdots+\left(k^{\alpha-1}-1\right) \delta_{k}\right] \\
\geq & 0,
\end{aligned}
$$

and thus the theorem follows.

Lemma 2 indicates that with a given $\mathbb{C}_{\text {ideal }}$, the battery pack's actual capacity delivery is maximized when the parallel cell strings are of similar strength. We have the following theorem on the upperbound of the battery pack's capacity delivery based on Lemma 2.

Theorem 1. Denote $\mathbb{C}_{\mathrm{rc}}^{*}$ as the maximum capacity delivery of the battery pack, then

$$
\mathbb{C}_{\mathrm{rc}}^{*} \leq\left(V \cdot I_{C} / P\right)^{\alpha-1}\lfloor n / m\rfloor^{\alpha-1} \mathbb{C}_{\text {ideal }}^{\mathrm{dp}} .
$$

where $\mathbb{C}_{\mathrm{ideal}}^{\mathrm{dp}}$ is the idealized capacity delivery identified by CSR.

Proof. From Lemma 2, we know the battery pack's capacity delivery is maximized when (i) its idealized capacity delivery $\mathbb{C}_{\text {ideal }}$ is maximized; (ii) the maximum number of cell strings are formed (i.e., $k$ is maximized) and they deliver the same capacity. As CSR identifies the configuration with maximum capacity delivery when the cells are ideal, we know

$$
\mathbb{C}_{\text {ideal }} \leq \mathbb{C}_{\text {ideal }}^{\text {dp }}
$$

Furthermore, it is clear that

$$
k \leq\lfloor n / m\rfloor .
$$

The theorem follows by combining these with Lemma 2.

Next we consider the lower bound of the actual capacity delivery of the configuration identified by CSR, consisting of $k^{\mathrm{dp}}$ parallel strings. Intuitively, its capacity delivery is minimized when we sequentially use the $k^{\mathrm{dp}}$ strings to support the load instead of discharge them in parallel, resulting 


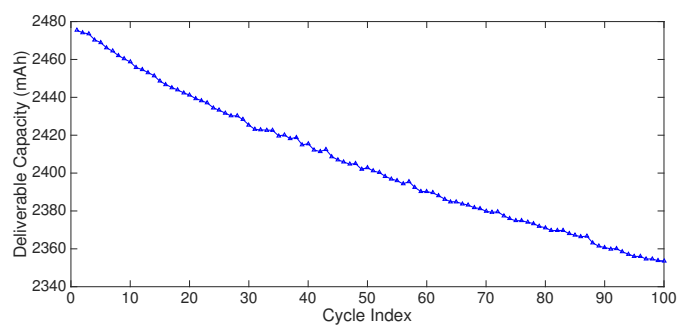

Figure 13: Cells' capacity fading over cycling.

in the largest possible discharge current of individual cells (i.e., $\frac{P}{V}$ ).

Theorem 2. For the configuration identified by CSR, its deliverable capacity $\mathbb{C}_{\mathrm{rc}}^{\mathrm{dp}}$ is minimized when the cell strings are used to support the load sequentially, specifically,

$$
\mathbb{C}_{\mathrm{rc}}^{\mathrm{dp}} \geq\left(V \cdot I_{C} / P\right)^{\alpha-1} \sum_{i=1}^{k^{\mathrm{dp}}} C_{i}=\left(V \cdot I_{C} / P\right)^{\alpha-1} \mathbb{C}_{\mathrm{ideal}}^{\mathrm{dp}}
$$

Finally, combining Theorem 1 and 2 leads to the following theorem on the near-optimality of CSR in capacity delivery.

Theorem 3. CSR achieves near-optimal capacity delivery of the battery pack, specifically,

$$
\frac{\mathbb{C}_{\mathrm{rc}}^{d p}}{\mathbb{C}_{\mathrm{rc}}^{*}} \geq \frac{\left(V \cdot I_{C} / P\right)^{\alpha-1} \mathbb{C}_{\mathrm{ideal}}^{\mathrm{dp}}}{\left(V \cdot I_{C} / P\right)^{\alpha-1}\left\lfloor\frac{n}{m}\right\rfloor^{\alpha-1} \mathbb{C}_{\text {ideal }}^{\mathrm{dp}}}=\frac{1}{\left\lfloor\frac{n}{m}\right\rfloor^{\alpha-1}} .
$$

Furthermore, we observe that (i) Theorem 3 is tight as the equality establishes when the configuration identified by CSR skips no cells and all the strings have the same capacity delivery; (ii) the configuration identified by CSR approaches the optimal case with smaller Peukert coefficient $\alpha$.

\section{SALIENT PROPERTIES OF CSR}

- CSR Alleviates Cell Imbalance in Long Run CSR is motivated by the commonly found cell imbalance in battery packs. Actually, besides improving the battery pack's capacity delivery during individual discharge cycles, CSR also reduces the cell imbalance in the pack over extended operation cycles. This is because (i) the capacity of cells decreases over their cycling process as our measurements shown in Fig. 13; (ii) with CSR, the weaker cells have higher chance to rest when compared to the stronger cells, leading to slower cycling and thus slower capacity fading.

- CSR is not Confined to JPL-Type Battery Packs The core operation of CSR is the DP-based cell skipping, which is based on the sequential properties of cells in JPLtype battery packs. Here we would emphasize that the sequential properties of cells are not only confined to JPL-type battery packs, but are shared by, to the best of our knowledge, all reconfigurable battery packs with a single pair of power buses (e.g., $[16,23])$. This is because these sequential properties are imposed by the physical laws - the physical direction of discharge current leads to the intra-string sequential property and the physical protection of cells from short leads to the inter-string sequential property.

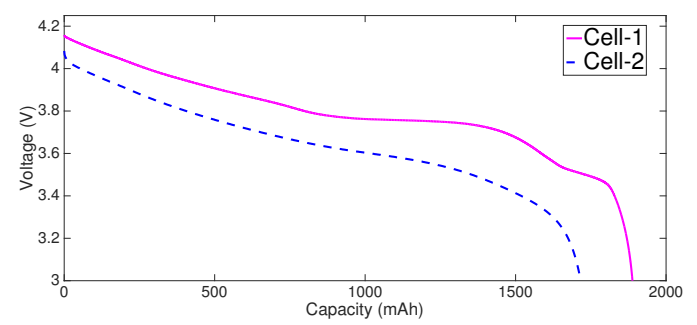

Figure 14: Exemplary discharge traces.

Table 1: Default emulation settings.

\begin{tabular}{|c|c|c|c|}
\hline Pack Scale & Peukert Coeff. & String Size & Current \\
\hline \hline 1,000 & 1.2 & 15 & $5 C$ \\
\hline
\end{tabular}

- CSR Goes Beyond Peukert's Law Peukert's law is classic for representing the relationship between the discharge rate and cells' capacity delivery, especially for constant discharge rate as in the system model shown in Fig. 7. However, it is known that the accuracy of Peukert's law degrades when the discharge rate varies over time [29]. Fortunately, CSR still applies to such variable discharge rates scenarios because the DP-based cell skipping assumes ideal cells in the battery pack and thus is not affected by variable discharge rates. The accuracy of Peukert's law, however, does affect the performance analysis presented in Sec. 5.3.

\section{EVALUATION}

We have evaluated CSR by emulating large-scale battery packs with empirically collected cell discharge traces. We have also investigated the sensitivity of CSR over cell imbalance degree via Monte Carlo simulations.

\subsection{Trace-Driven Emulation}

We collect 40 rechargeable Lithium-ion cells, fully charge and then discharge them individually with $1 \mathrm{C}$ rate with the NEWARE battery tester, which allows not only high accuracy charge/discharge control but also fine-grained logging of the experiments. Fig. 14 shows two exemplary thusobtained discharge traces and the lab settings for the measurements are shown in Fig. 15. Fig. 16 summarizes the capacity delivery of these cells ascendingly. We emulate JPLtype battery packs with these traces - each cell in the pack are randomly emulated to be one of these 40 cells in Fig. 16. Table 1 lists the default settings unless specified otherwise. The emulator is implemented with Matlab. The reported results are averaged over 500 runs.

We also emulate non-reconfigurable battery packs in which no cells could be skipped - the first $m$ cells in the pack form the first string, the second $m$ cells form the second string, etc. The Oracle capacity delivery calculated according to Theorem 1 is also explored for comparison.

\subsubsection{Impact of Battery Pack Scale}

Fig. 17 plots the capacity delivery of battery packs consisting of 200-1, 000 cells. The JPL-type battery pack with CSR delivers more capacity than the non-reconfigurable packs for all explored cases, especially for large battery packs. For example, CSR delivers 23,383mAh more capacity than the non-reconfigurable case for 1,000 -cell battery packs. This indicates CSR is particularly desirable for large-scale bat- 


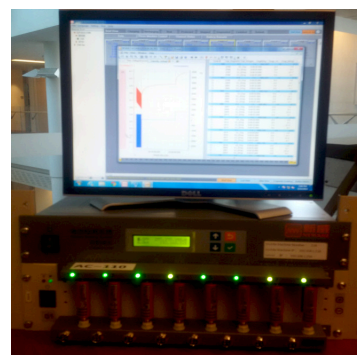

Figure 15: Lab settings for discharge measurements.

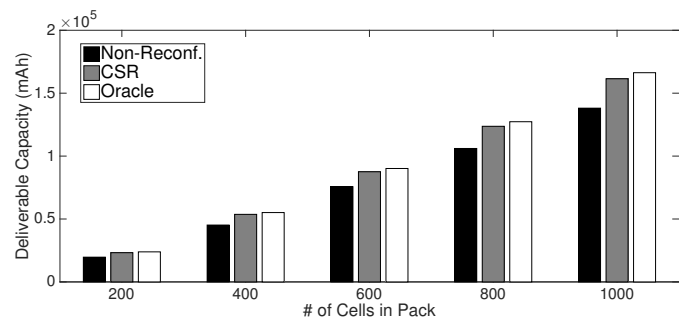

Figure 17: Capacity delivery with various battery pack scales.

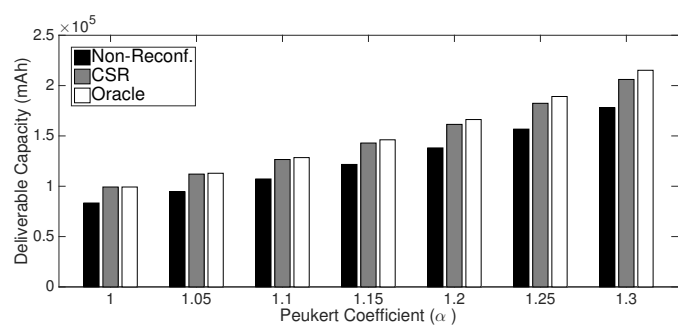

Figure 18: Capacity delivery with various Peukert coefficients.

tery systems such as EVs. Also, CSR achieves close capacity delivery to the Oracle solution - although the gap between CSR and Oracle increases with larger battery pack scales, a performance ratio of $97.1 \%$ is achieved even for the 1,000 cell battery packs.

\subsubsection{Impact of Peukert Coefficient}

Fig. 18 shows the capacity delivery with various Peukert coefficients, from which two observations can be made. First, compared to the non-reconfigurable case, the advantage of CSR is pronounced for cells with small Peukert coefficients. For example, the capacity delivery is improved by $19 \%$ with an $\alpha$ of 1.00 , which reduces to $\approx 16 \%$ with $\alpha=1.30$. Second, CSR achieves the Oracle capacity delivery when cells are ideal (i.e., when $\alpha=1$ ), and its gap to the Oracle solution increases as $\alpha$ increases. However, even with $\alpha=1.30$, CSR still achieves a performance ratio of $96 \%$ when compared to Oracle. Both these observations indicate that CSR works better for cells with smaller Peukert coefficient, which is also the direction of battery development as cells with smaller $\alpha$ indicates good efficiency and less loss [2].

\subsubsection{Impact of Load Required Discharge Rate}

Fig. 19 plots the capacity delivery with various load required current. CSR outperforms the non-reconfigurable battery packs especially with smaller load required discharge

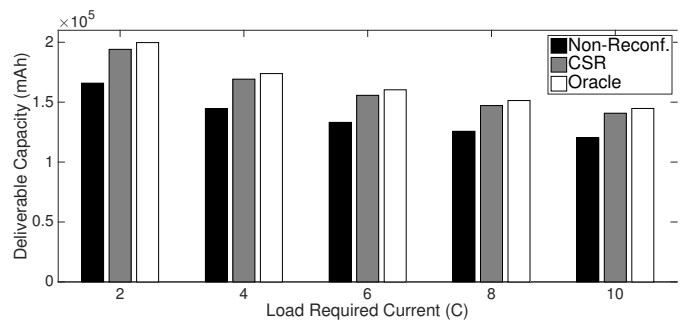

Figure 19: Capacity delivery with various load required discharge rates.

Table 2: Number of parallel strings.

\begin{tabular}{|c||c|c|c|c|c|}
\hline String Size $(m)$ & $\mathbf{5}$ & $\mathbf{1 0}$ & $\mathbf{1 5}$ & $\mathbf{2 0}$ & $\mathbf{2 5}$ \\
\hline \hline CSR & 192.35 & 93.83 & 61.62 & 45.71 & 36.27 \\
\hline Maximum $\left(\left\lfloor\frac{n}{m}\right\rfloor\right)$ & 200 & 100 & 66.67 & 50 & 40 \\
\hline \hline Skipped Cells & 38.25 & 61.70 & 75.70 & 85.80 & 93.25 \\
\hline
\end{tabular}

rates. For example, about $20,577 \mathrm{mAh}$ more capacity is delivered with a $10 \mathrm{C}$ load current, which increases to $28,238 \mathrm{mAh}$ when the load current reduces to $2 \mathrm{C}$. This is because larger load currents pronounce the rate-capacity effect, which is ignored by the DP-based cell skipping. This way, the advantage of CSR diminishes as the load current increases. However, CSR improves the capacity delivery by $\approx 17 \%$ even with $10 \mathrm{C}$ load current. CSR achieves a performance ratio of $\approx 97 \%$ when compared to the Oracle, agreeing with Fig. 17 .

\subsubsection{Number of Formed Strings}

Clearly, skipping cells from discharge leads to fewer cell strings when compared to using all the cells to support the load. Table 2 lists the average number of parallel strings formed by CSR with various load required string sizes. We also list the maximum number of parallel strings for comparison (i.e., $\left\lfloor\frac{n}{m}\right\rfloor$ ). Not surprisingly, fewer strings are formed with CSR. Multiplying the reduction in the number of formed strings and the corresponding required string size, we can see more cells are skipped when the load requires longer strings. For example, $(40-36.27) \times 25=93.25$ cells are skipped on average when 25-cell strings are required, while only $(200-192.35) \times 5=38.25$ cells are skipped for the 5 -cell string case. Again, this indicates the selective cell skipping is especially important for high voltage load applications (i.e., requiring longer cell strings) such as EVs.

\subsubsection{Skipped Cells Distribution}

CSR is inspired by the observation that selectively skipping weak cells from discharge may improve the battery 


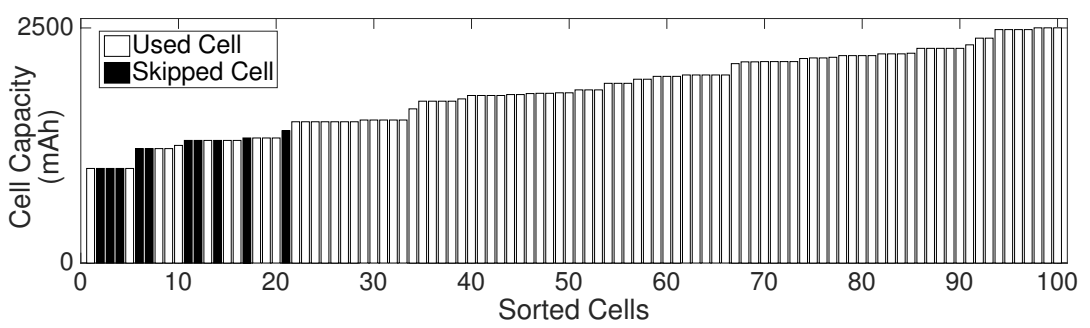

Figure 20: CSR skips weak cells but not necessary the weakest ones.

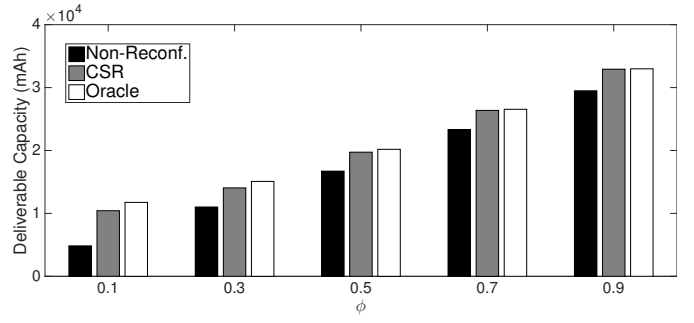

Figure 21: Sensitivity of CSR over cell imbalance.

pack's capacity delivery; however, CSR does not simply skip the weakest cells. To demonstrate the different between CSR and the greedy cell skipping, Fig. 20 plots the skipped cells in an emulated 100-cell battery pack. ${ }^{5}$ By ascendingly sorting the cells according to their respective deliverable capacity, we find although the skipped cells by CSR are clustered at the low capacity spectrum, they are not simply the weakest ones. Specifically, for this particular battery pack, CSR identifies a configuration with $9,849 \mathrm{mAh}$ deliverable capacity, while that with the greedy approach delivers only 9, 509mAh.

\subsection{Sensitivity Analysis over Cell Imbalance}

CSR is inspired by the wide existence of cell imbalance in battery packs. We next investigate the sensitivity of CSR over cell imbalance degree. To capture the cell imbalance degree, we introduce a control parameter $\phi(\phi \in[0,1])$ and randomly generate the cell capacity under $1 \mathrm{C}$ rate in the range of $[\phi, 1] \times c_{0}$, where $c_{0}$ is the rated capacity of cells and specifically, $2,300 \mathrm{mAh}$ in our settings. This way, a smaller $\phi$ indicates higher cell imbalance in the battery pack. We apply CSR to JPL-type battery packs, and again, compare it to the non-reconfigurable battery packs and the Oracle solution. Fig. 21 plots the collected results averaged over 500 runs. Not surprisingly, the advantage of CSR over the nonreconfigurable case is pronounced with larger cell imbalance degree, e.g., about $94 \%$ improvement with a $\phi$ of 0.1 . Note this small $\phi$ can be interpreted as the case that certain cells fail over usage, which is commonly found in practice $[3,7]$. CSR approaches the Oracle as cells become more balanced.

\section{RELATED WORK}

In general, existing studies of reconfigurable battery packs can be classified into two categories: offering reconfigurability and exploiting reconfigurability.

\subsection{Design of Reconfigurable Battery Packs}

The system reconfigurability is achieved by adding switches

\footnotetext{
${ }^{5}$ The relatively small battery pack scale is to ease the ob-
} servation in Fig. 20. into the battery pack and thus increases system complexity. Much research effort has been devoted to achieve high reconfigurability with low system complexity. Kim et al. [26] proposed a reconfigurable battery pack design in which each cell is equipped with two switches - one for connecting the cell to the load and the other for skipping the cell. With this design, any subset of cells can be sequentially connected in series, but no parallel strings can be formed. The JPLtype battery pack [11] considered in this paper allows for forming multiple parallel strings by equipping four switches to each cell. Increasing the per-cell switches to six, a reconfigurable battery pack supporting multiple outputs was proposed in [23].

\subsection{Reconfiguration-Assisted Optimization}

The other category of existing work on reconfigurable battery packs focuses on exploitation of the offered reconfigurability to improve the battery performance. For example, a reconfiguration algorithm by matching the supplied and required voltages, and thus reducing the energy loss due to voltage regulation, was presented in [18]. The trade-off between cycle efficiency and capacity utilization of the battery pack was explored in [27]. The reconfiguration-assisted charging of battery packs was explored in [21].

Exploiting system reconfigurability to mitigate cell imbalance has also received attentions from the community [19, 26]. For example, Ci et al. [16] used reconfiguration to enhance the energy utilization of the battery pack via a Lagrangian relaxation. He et al. used a generalized graph model to mitigate the cell imbalance. In this paper, we advance the state-of-the-art by presenting a concrete case study of mitigating cell imbalance with the limited reconfigurability offered by JPL-type battery packs.

\section{CONCLUSION}

In this paper, we presented a case study of mitigating the cell imbalance in battery packs via system reconfiguration. We abstracted the problem of identifying the configuration with maximum capacity delivery to a cell-selection problem based on two sequential properties of cells in the battery pack. Then, we presented CSR, a reconfiguration algorithm that identifies a near-optimal configuration with a dynamic programming approach. Our trace-driven emulation based on 40 Lithium-ion cells shows CSR achieves the optimal capacity delivery when the cell imbalance is low and improve the battery pack's capacity delivery by up to $94 \%$ in case of high imbalance.

Acknowledgment: The work reported in this paper was supported in part by NSF under Grants CNS-1329702 and CNS-1446117, and LG Chemistry. 


\section{REFERENCES}

[1] A Guide to Understanding Battery Specifications. http://web.mit.edu/evt/summary_battery_ specifications.pdf.

[2] Battery University. http://batteryuniversity.com/ learn/article/calculating_the_battery_runtime.

[3] Honda Civic Hybrid Battery Failure. http://www. aa1car.com/library/honda_civic_hybrid_battery.htm.

[4] Microsoft Aims for Smartphones That Run for a Week. http://www.technologyreview.com/news/528201/.

[5] Peukert's Law Tutorial. https: //www.easycalculation.com/physics/classical-physics/ learn-battery-capacity-discharge-time.php.

[6] Reconfigurable Battery Packs. http://arpa-e.energy. gov/q=arpa-e-projects/reconfigurable-battery-packs.

[7] Tesla Battery Failures Make Bricking a Buzzword. http://www.nytimes.com/2012/03/04/automobiles/ Tesla-Battery-Failures-Make-Bricking-a-Buzzword. html.

[8] Tesla Model S Specification. http://www.teslamotors. com/support/model-s-specifications.

[9] The Basics of Arc Flash. https://www.geindustrial.com/sites/geis/files/gallery/ The-Basics-of-Arc-Flash-Article_GE_Industrial Solutions_0.pdf.

[10] Understanding "Arc Flash". https://www.osha.gov/dte/grant_materials/fy07/ sh-16615-07/arc_flash_handout.pdf.

[11] M. Alahmad, H. Hess, M. Mojarradi, W. West, and J. Whitacre. Battery switch array system with application for JPL's rechargeable micro-scale batteries. Journal of Power Sources, 177(2):566 - 578, 2008.

[12] D. Andrea. Battery management systems for large Lithium-ion battery packs. Artech House, 2010.

[13] A. Badam, R. Chandra, J. Dutra, A. Ferrese, S. Hodges, P. Hu, J. Meinershagen, T. Moscibroda, B. Priyantha1, and E. Skiani. Software defined batteries. In SOSP'15, 2015.

[14] B. Chai, J. Chen, Z. Yang, and Y. Zhang. Demand response management with multiple utility companies: A two-level game approach. IEEE Transactions on Smart Grid, 5(2):722-731, March 2014.

[15] S. Chandra, D. Gayme, and A. Chakrabortty. Coordinating wind farms and battery management systems for inter-area oscillation damping: A frequency-domain approach. Power Systems, IEEE Transactions on, 29(3):1454-1462, May 2014.

[16] S. Ci, J. Zhang, H. Sharif, and M. Alahmad. Dynamic reconfigurable multi-cell battery: a novel approach to improve battery performance. In APEC'12, 2012.

[17] L. H. Goldberg. Battery cell balancing for improved performance in EVs. Electronic Products Magazine, 2011.

[18] L. He, L. Gu, L. Kong, Y. Gu, C. Liu, and T. He. Exploring adaptive reconfiguration to optimize energy efficiency in large-scale battery systems. In $R T S S^{\prime} 13$, 2013.

[19] L. He, Y. Gu, C. Liu, T. Zhu, and K. G. Shin. SHARE: SoH-aware reconfiguration to enhance deliverable capacity of large-scale battery packs. In ICCPS'15, 2015.

[20] L. He, E. Kim, and K. G. Shin. *-aware charging of lithium-ion battery cells. In ICCPS'16, 2016.

[21] L. He, L. Kong, S. Lin, S. Ying, Y. Gu, T. He, and C. Liu. Reconfiguration-assisted charging in large-scale Lithium-ion battery systems. In ICCPS'14, 2014.

[22] S. He, J. Chen, F. Jiang, D. K. Y. Yau, G. Xing, and Y. Sun. Energy provisioning in wireless rechargeable sensor networks. IEEE Transactions on Mobile Computing, 12(10):1931-1942, Oct 2013.

[23] H. Kim and K. G. Shin. On dynamic reconfiguration of a large-scale battery system. In RTAS'09, 2009.

[24] H. Kim and K. G. Shin. Dependable, efficient, scalable architecture for management of large-scale batteries. In $I C C P S^{\prime} 10,2010$.

[25] T. Kim, W. Qiao, and L. Qu. Series-connected self-reconfigurable multicell battery. In $A P E C^{\prime} 11$, 2011.

[26] T. Kim, W. Qiao, and L. Qu. A series-connected self-reconfigurable multicell battery capable of safe and effective charging/discharging and balancing operations. In APEC'12, 2012.

[27] Y. Kim, S. Park, Y. Wang, Q. Xie, N. Chang, M. Poncino, and M. Pedram. Balanced reconfiguration of storage banks in a hybrid electrical energy storage system. In ICCAD'11, 2011.

[28] G. Motors. Latest chevy volt battery pack and generator details and clarifications, 2016.

[29] N. Omar, P. V. d. Bossche, T. Coosemans, and J. V. Mierlo. Peukert revisited: Critical appraisal and need for modification for Lithium-ion batteries. Energies, 6(11):5625-5641, 2013.

[30] D. Raychev, Y. Li, and W. Shi. The seventh cell of a six-cell battery. In WEED'11, 2011.

[31] K. Vatanparvar and M. A. A. Faruque. Battery lifetime-aware automotive climate control for electric vehicles. In $D A C^{\prime} 15,2015$.

[32] H. Visairo and P. Kumar. A reconfigurable battery pack for improving power conversion efficiency in portable devices. In ICCDCS'08, 2008. 